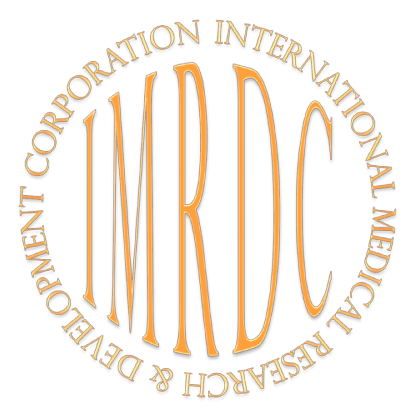

\title{
Cardiology
}

\section{Spatial Synchronization of Hemodynamics and Metabolism in Norm}

\author{
Alexander G. Kruglov, PhD, ScD*; Valery N. Utkin; \\ Alexander Yu. Vasilyev, PhD, ScD; Andrey A. Kruglov, PhD \\ Central Research Institute of Radiation Diagnostics \\ Moscow, the Russian Federation
}

\begin{abstract}
Synchronization of the curves of central and peripheral hemodynamics, according to the data obtained during catheterization, showed the intersection points of the graphical pressure curves, which were shown in previous works. The three-chamber block of the ventricles (with leading role of left ventricle) in the isometric contraction phase $(D(Q))$ generates three wave impulses: 1) retrograde impulse of coronary sinus directed to the zone of the integral combination (hemodynamics, metabolites of metabolic zones, hormones) and partial interference of venous flows coming to right atrium (end section of systemic circulation); 2) anterograde impulse of right ventricle, which heads to the exchange zone of the lung (initial section of pulmonary circulation) and outrunning of the "venous bolus"; 3 ) anterograde impulse of left ventricle, ahead of the "arterial bolus" (initial section of systemic circulation), leaving along Ao into the vascular bed to the exchange zones of peripheral organs.

We have interpreted intersections of hemodynamic curves as "zone of temporal equalization of pressure" (ZTEP), both in the heart and in remote topographic zones. Based on the results of previous works, we built separate ZTEP plots for the high- and lowenergy phases of the cardiac cycle (CC), relative to cardiac mean integral pressure (CMIP). The genesis and development of key points of the low-energy phase are considered. We believe that the distributed ZTEP matrix created in the vascular bed during each CC, consisting of short-term synchronous equal-sized pressor structures interfering with the aorta wave impulse, has a regulatory effect on the $\mathrm{CC}$ phase sequence, the hemodynamics of organs (including peripheral resistance), and metabolism. The systemic distributed ZTEP association - a formed CMIP - is a synchronized (between ZTEPs, with the phases of CC, and electrocardiography), high-speed, information-regulatory structure of the interaction of hemodynamics and metabolism of both central and peripheral organs, which is involved in the control and regulation of homeostasis as a whole. (International Journal of Biomedicine. 2020;10(1):24-28.)
\end{abstract}

Key Words: cardiosynchronization $\bullet$ pulse wave $\bullet$ cardiac hemodynamics $\bullet$ hemostasis

\section{Abbreviations}

Ao, aorta; CC, cardiac cycle; CBF, cerebral blood flow; CS, coronary sinus; Cat, catalase; CMIP, cardiac mean integral pressure; $\mathbf{E F}$, ejection fraction; ECG, electrocardiography; Er, erythrocyte; F-n, fibrinogen; IC, integral curve; IVC, inferior vena cava; $\mathbf{L A}$, left atrium; LV, left ventricle; MV, mitral valve; PC, pulmonary circulation; PEP, pressure equalization point; P-n, total protein; PV, pulmonary valve; PT, pulmonary trunk; RA, right atrium; RHV, right hepatic vein; PW, pulse wave; Plas, plasma; Prot, protein; RV, right ventricle; SC, systemic circulation; SVC, superior vena cava; SS, sigmoid sinus; SAH, stabile arterial hypertension; TV, tricuspid valve; TP, trigger point; ZTEP, zone of temporal equalization of pressure.

\section{Materials and Methods}

The aim of this study was to determine the mechanisms of synchronization of zones of temporal equalization of pressure (ZTEPs) obtained during the construction of graphs of central and peripheral hemodynamics.

ZTEPs in the points of intersection of pressure curves (calculated by average values) on the graphs that we obtained during catheterization of vessels in the normal state ${ }^{(1-5)}$ denote short periods of equality of pressure indicators in blood sampling points. The intersection of the graphical pressure curves for a particular subject is a PEP (pressure equalization

*Corresponding author: Alexander G. Kruglov, PhD, ScD. Central Research Institute of Radiation Diagnosis. Moscow, the Russian Federation.E-mail: krag48@mail.ru 
point), which does not have a range of confidence interval for coefficient value (as opposed to average values), which means a short-term alignment of pressure indicators at two or more exchange entry/exit exchange zones of two or more organs. In the graphs at the intersections of the curves, we registered point coincidence and pressure equalization for hemodynamic curves synchronized in time with the CC phase and ECG, which together constitute a distributed system.

\section{Results and Discussion}

The tables we gave earlier ${ }^{(6,7)}$ contain the data of a correlation analysis between the parameters of metabolism and hemodynamics obtained at the points of blood sampling during catheterization in all organs we examined. We believe that each topographically stable point of catheterization, a complete set of which is presented in the form of "pressure curves" at all stages of pressor dynamics, is synchronous to the phase of metabolism (metabolic activity) in the corresponding organ at the time the initial data were obtained (hemodynamics, biochemistry, hormones). As an example, we present the data of correlations obtained at the input (Ao) and output (SS) from the cerebral blood flow of metabolites (i.e. a dynamic summary structure of brain metabolic connections, without impurities of extracranial blood) (Tables 1 and 2).

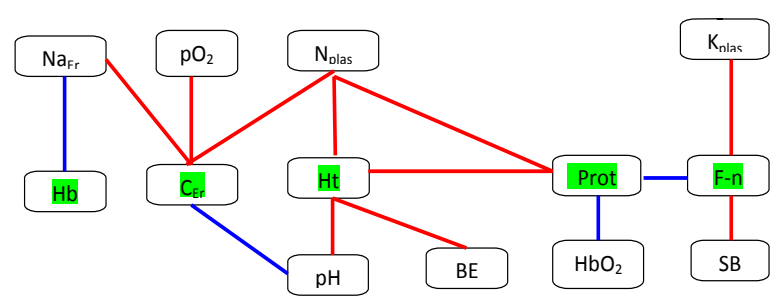

Scheme 1. The input in the CBF system (control group) - Ao
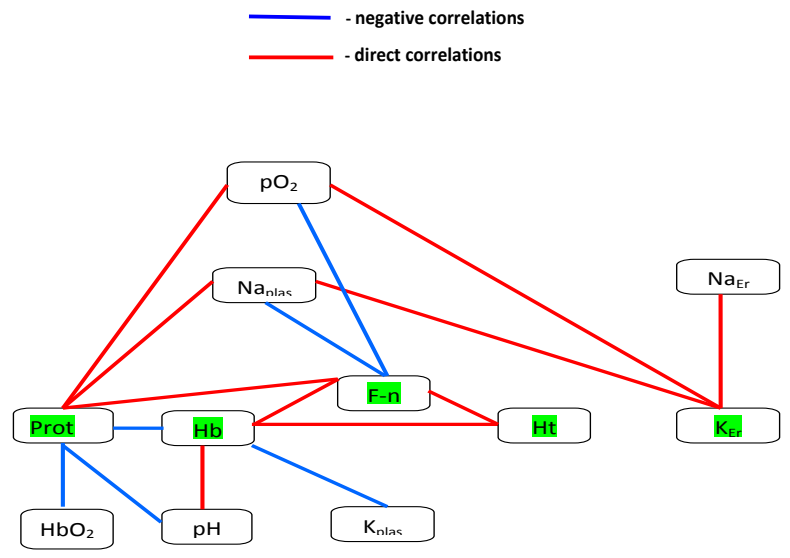

Scheme 2. The output from the CBF system (control group) - SS

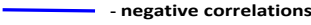

- direct correlations

As the "structural" parameters, we chose a number of indicators whose concentration does not change throughout one CC (F-n, P-n, $\mathrm{K}_{\mathrm{Er}}$, and other). For a comparative analysis, we present similar indicators obtained by catheterization in patients with $\mathrm{SAH}^{(7)}$ (Tables 3 and 4).

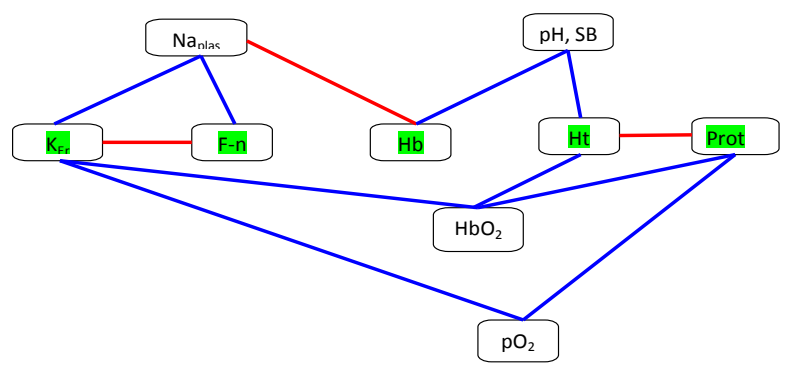

Scheme 3. The input in the CBF system (SAH) - Ao
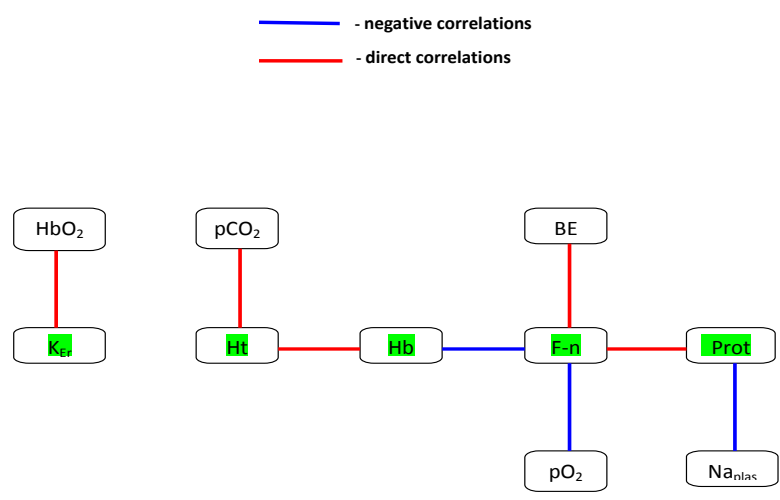

Scheme 4. The output from the CBF system (SAH) - SS

We note radical differences from the norm in the entire set of correlation relationships (i.e. the structures of cerebral metabolism, both at the entrance and at the exit from the system of cerebral blood flow in SAH).

The differences we found in the mechanisms (including metabolic) of maintaining stable cerebral blood flow in normal conditions and hypertension were significant. We presented our analysis ${ }^{(7)}$ of the differences between the norm and SAH earlier. Here, we emphasize the synchronism of hemodynamic and metabolic organ processes at the informational point when receiving data by catheterization.

In other words, the data obtained during catheterization made it possible to obtain not only certain indicators, but also (through a set of correlation connections) the ability to build synchronous graphical portraits of hemodynamics and intraorgan metabolism (similar to the above for cerebral blood flow) for each organ examined.

Our data ${ }^{(1-5)}$ made it possible to construct separate ZTEP plots of the high- and low-energy phases of $\mathrm{CC}$ relative to each other and the average integral pressure of $\mathrm{CC}$ as a universal rhythmic cycle that sets the main parameters of the frequency and intensity of all hemodynamic wave processes in the body (Fig.1).

Damping and dissociation of the stroke energy of the aortic flow wave structures and targeted selection of segments 


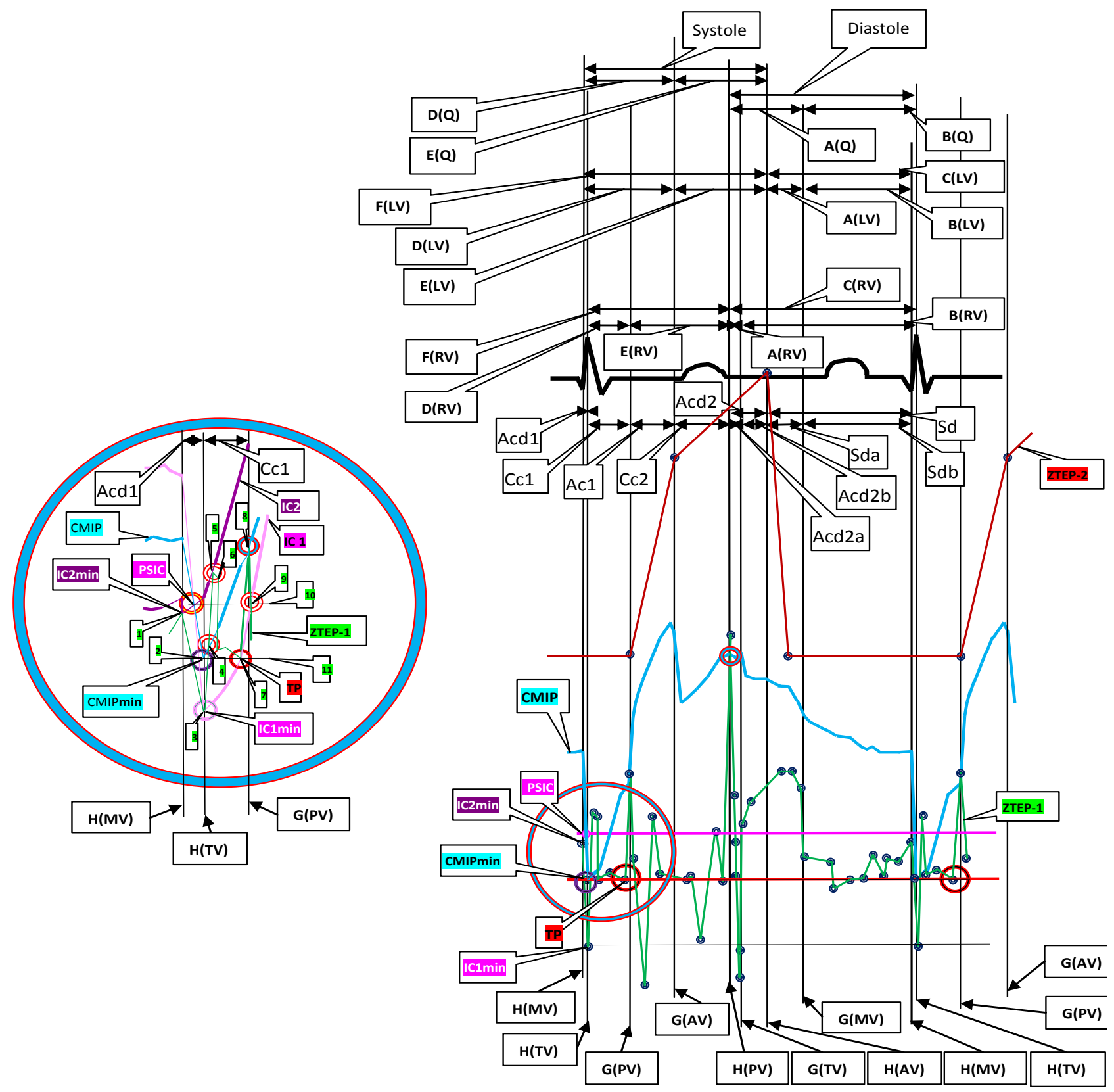

\begin{tabular}{|c|c|}
\hline & Fig.1. ZTEP of the high- and low-energy phases of CC \\
\hline $\begin{array}{l}\text { A(Q) - isometric ventricular relaxation } \\
\text { B(Q) - actual ventricular diastole } \\
\text { C(LV) - LV diastole } \\
\text { A(LV) - isometric LV relaxation } \\
\text { B(LV) - actual LV diastole } \\
\text { C(RV) - RV diastole } \\
\text { A(RV) - isometric RV relaxation } \\
\text { B(RV) - actual RV diastole } \\
\text { D(Q) - isometric ventricular contraction } \\
\text { E(Q) - actual ventricular systole } \\
\text { F(LV) - LV systole } \\
\text { D(LV) - isometric LV contraction } \\
\text { E(LV) - actual LV systole } \\
\text { F(RV) - RV systole } \\
\text { D(RV) - isometric RV contraction } \\
\text { E(RV) - actual RV systole } \\
\text { G(AV) - opening of AV } \\
\text { H(PV) - closing of PV } \\
\text { G(TV) - opening of TV } \\
\text { H(AV) - closing of AV } \\
\text { G(MV) - opening of } M V \\
\text { H(TV) - closing of TV } \\
\text { H(MV) - closing of } M V \\
\text { G(PV) - opening of } P V \quad H(P V) \text { - closing of PV }\end{array}$ & 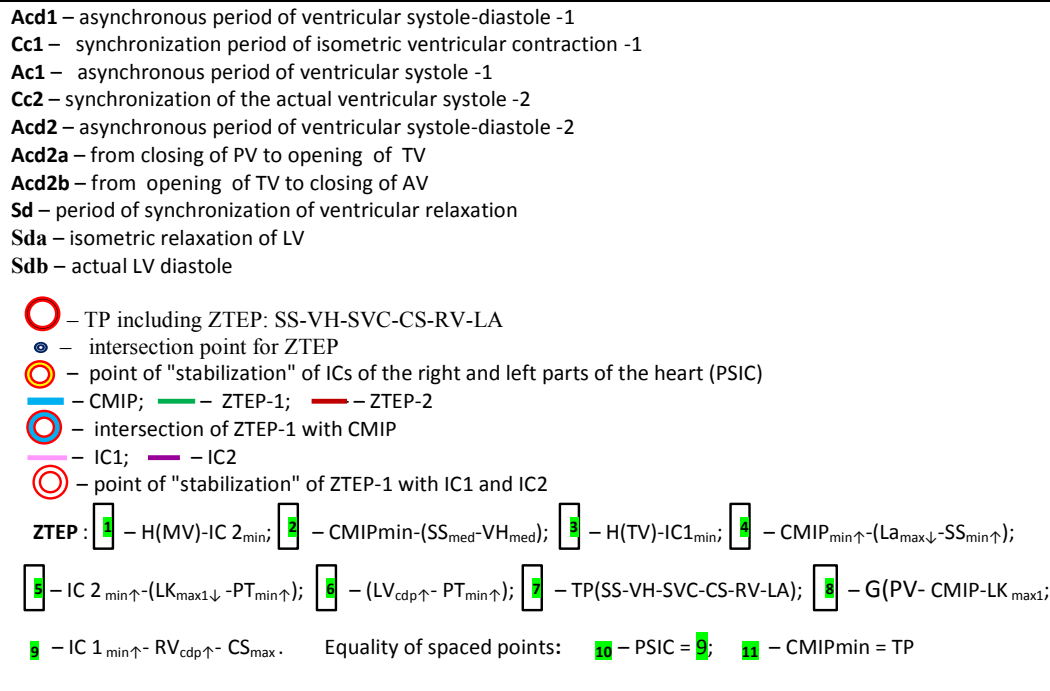 \\
\hline
\end{tabular}


(spheroid sectors) of the arterial bolus directed to the exchange zones are ensured by evolutionarily determined angles of departure from Ao and further branching of the great vessels conducting the impulse and then the blood substrate from Ao to each organ of the body.

For example, Truncus coeliacus, departing from the aorta at a right angle, is divided into A. lienalis and $\mathrm{A}$. hepatica, which depart from it at sharp angles, transforming the propagation and influence of the impulse wave to the exchange zones, adapting (specializing) PW for each organ. Previously, we described the damping effect of SS for the exchange fields of brain tissue..$^{(7)}$ Also, we presented data ${ }^{(6,8)}$ on the mechanism of the influence of PW propagating along the vascular wall and outrunning of the movement of the blood substrate along the vascular bed. In other words, the angular characteristics of the "Ao-main artery" relationship determine the segment of the aortic spheroid, speed, and volume of the arterial bolus fragment, targeted from the aortic bed to the exchange zones of organs. That is, each organ receives an individual wave impulse specific to it ("information packet") at the entrance to the organ's exchange zone, with the same biochemical composition as the arterial blood that is coming.

In the venous sectors of $\mathrm{SC}$ and $\mathrm{PC}$, hemodynamic gradients ("organ-heart") form boost pressure at the exit from organs (exchange zones). In the arterial sector, hemodynamic gradients ("heart-organ") are involved in adaptive changes in blood vessels (address distribution of aortic spheroid segments) at the entrance to the exchange zone to optimize the intake of blood substrate brought by the arterial bolus.

The ZTEPs on the graphs indicate PEP characterizing topographic stability, equality of pressor indices, and shortterm synchronism in time and $\mathrm{CC}$ phase, making it possible to construct an objective picture of metabolism in PEP coincidence organs.

In other words, ZTEPs (PEPs) make it possible to obtain simultaneous, synchronous information about organ hemodynamics and the structure of intraorgan metabolism in a certain phase of CC for organs with coincident ZTEPs (PEPs). The sequential distribution of ZTEPs among the SC and PC hemodynamic vectors, synchronized with ECG and CC phases, makes it possible to construct a design for monitoring and regulating hemodynamic and metabolic homeostasis as a whole.

We consider it appropriate to divide the ZTEP routing into two routes, high- and low-energy, in accordance with the $\mathrm{CC}$ phases (on the graph: above and below CMIP). High-energy ZTEPs are points of pressure-mediated effects (through ductile walls at points of direct contacts with LV and Ao; ligamentous apparatus, for example, lig. arteriosum, connecting Ao and PT, etc.) of the dynamics of isometric transformation of the ventricles (RV/LV systole) and arterial wave impulse on RA, PT, LA and peripheral vessels (shown in the part of the graph above CMIP).

In this paper, we deem it appropriate to consider some ZTEPs related to the low-energy phase of CC. Each CC begins with IC-2min, attributable to the end of the previous $\mathrm{CC}$ with the closure of $\mathrm{MV}$ and the beginning of the asynchronous period of isometric contraction of the ventricles (Acd1) of the current CC.
During this period, against the background of the completion phase of the RV diastole and contraction of the RA appendage of the previous $\mathrm{CC}$, a period of isometric contraction of $\mathrm{LV}$ of the subsequent $\mathrm{CC}$ begins, forming the CS impulse. This impulse, interfering with the RA appendage impulse, reaches RV (i.e. the final stages of the dynamics of the exchange zones of the brain (SVC) and liver (IVC) and other organs, participating in the creation of boost pressure in these zones).

In the middle of Acd1, a point of stabilization of the integral pressure curves (PSIC) of the right and left heart (IC-1, IC-2) is formed. PSIC is a point of temporary pressure equalization at the SC inlet and outlet, described in a previous work. ${ }^{(5)}$ We believe that PSIC: 1) determines the sequence of triggering phases of the isometric contractions of the ventricles (RV after LV); and 2) determines the level of perfusion pressure in the brain, liver and lung after the opening of PV. The mechanism of synchronization of the "ventricular block" in the period of the formation of the spheroids of RVEF (current cycle) and LVEF (the end of the previous cycle, which influences the RV by the CS ejection impulse) is initiated in PSIC.

The next ZTEP point in the Acd1 period is CMIPmin, which coincides with ZTEP at the pressor level of exit from the brain and liver exchange zones (SSmed-VHmed). It creates equal hemodynamic conditions in the exchange zones (including equal pressure levels), forming hemodynamic parameters of the next stage (TP).

The Acd1 period ends with IC-1min, attributable to the closure of TV and the beginning of the synchronization period of the isometric ventricular contraction (Cc-1). IC-1 min is characterized by the creation of a base pressure in $\mathrm{RV}$ at the time TV closes (VDcdd).

The following is the period of synchronization of isometric ventricular contraction $(\mathrm{Cc} 1)$, characterized by: 1) involvement of RV to CS impulse formation in $\mathrm{LV}$, and 2) complete isolation of the three-chamber block of the ventricles (except CS) from the $\mathrm{SC} / \mathrm{PC}$ collectors of variable capacitance. Thus, the CS impulse is twice corrected during the phase of the isometric contraction of the ventricles $(\mathrm{D}(\mathrm{Q})$, interfering with the RA appendage systole and the isometric contraction of $\mathrm{RV}$, which during the period $\mathrm{Cc} 1$ is "superimposed" on the isometric contraction of LV. During this period, due to the systolic suction function of the ventricles and the dynamics of pressure gradients in the variable capacities of the venous SC segment and arterial PC segment, TP is formed, uniting SS-VH-SVC-CS-RV-LA in ZTEP. This TP is synchronized (vertical) with Aomin and PTmin. ${ }^{(2-4)}$ TP determines equal hemodynamic conditions (including boost pressure at the exit from the organ), at all the indicated ZTEP points, ensuring equal peripheral resistance for the incoming Ao wave impulse and the spheroid of the arterial bolus in the corresponding exchange zones: brain, heart, lung, and liver. The main role of TP is to create the initial hemodynamic matrix for the upcoming CC, for which purpose the pressor indices at the exit from the SC and PC organ exchange zones are optimized, synchronizing and creating equal conditions in the microcirculatory bed of all exchange zones, adequate for the current homeostasis.

We believe that it is TP, completing the diastolic evolution of $\mathrm{CC}$, which is the beginning pressure level for 
starting high-energy processes of the right heart, initiating the following phases of $\mathrm{CC}$ : the opening of $\mathrm{PV}$, the beginning of LA systole (from the TP pressure level - boost pressure for pulmonary veins) and the subsequent $\mathrm{CC}$ sequence. In other words, the isometric contraction pressure of LV increasing during the Acd1 period forms CMIPmin, which at the end of the next period ( $\mathrm{Cc} 1)$, before the opening of PV, creates TP in the corresponding zones of the vascular bed. Thus, the diastole phase of the previous $\mathrm{CC}$ is completed, forming the base pressure in the venous section of the next CC.

We believe that during the period of isometric contraction of the ventricles ((D (Q)), along with others, the hemodynamic components of the next CC are formed: 1) the CS impulse, corrected by the RA systole and isometric RV contraction (Cc1), is involved in the formation of the boost pressure at the final exit stage from SC exchange zones (in RA); 2) PSIC, which determines the perfusion pressure levels of the SC (brain, liver, heart, and kidneys) and PC (lung) exchange zones after the opening of $\mathrm{PV}$ and initiating synchronization of the ventricular block during the formation of the spheroids of RVEF (current cycle) and LVEF (the end of the previous CC, affecting the RV by the CS impulse); 3) CMIPmin, creating equal hemodynamic conditions (including equal pressure levels from the exchange zones), defining the TP parameters, which structures the hemodynamic matrix of the next CC; and 4) IC-1min, which forms the RV base pressure at the time of TV closing, while isolating the "ventricular block" from the $\mathrm{SC} / \mathrm{PC}$ collectors of variable capacitance.

The distributed ZTEP system is formed by the universal, multidimensional structure of CMIP, ${ }^{(5)}$ changing the architectonics of the relationship between the venous capacitive vessels, arterial vessels, chambers of the heart and the vascular bed as a whole, creating targeted pressor impulses in the structure of systemic hemodynamics.

We believe that ZTEPs are short-term synchronized isometric pressor structures that exert a regulatory effect on the metabolism and hemodynamics of various organs, affecting peripheral resistance and interfering with the Ao impulse. The combination of several ZTEPs (for example, TP) can have a significant range of effects on hemodynamics, metabolism (see above) and the formation of subsequent hemodynamic structures

\section{Conclusion}

CMIP, forming the hemodynamics of the vascular bed as a whole, creates a distributed high-speed structure of short- term equilibrium hemodynamic formations (ZTEPs), affecting the phase sequence of $\mathrm{CC}$, peripheral resistance, and organ metabolism, as well as interacting with the Ao hemodynamic impulse. We believe that the systemic distributed ZTEP association - a formed CMIP - is a synchronized (between ZTEPs, with the phases of CC, and ECG), high-speed, information-regulatory structure of the interaction of hemodynamics and metabolism of both central and peripheral organs, which is involved in the control and regulation of homeostasis as a whole.

\section{Competing Interests}

The authors declare that they have no competing interests.

\section{References}

1. Kruglov AG, Utkin VN, Vasilyev AYu. Synchronization of Wave Flows of Arterial and Venous Blood with Phases of the Cardiac Cycle in Norm: Part 1. International Journal of Biomedicine. 2018;8(2):123-128.

2. Kruglov AG, Utkin VN, Vasilyev AYu, Kruglov AA. Synchronization of Wave Flows of Arterial and Venous Blood and Phases of the Cardiac Cycle with the Structure of the Peripheral Pulse Wave in Norm: Part 2. International Journal of Biomedicine. 2018;8(3):177-181.

3. Kruglov AG, Utkin VN, Vasilyev AYu, Kruglov AA. Synchronization of Wave Flows of Arterial and Venous Blood and Phases of the Cardiac Cycle with the Structure of the Peripheral Pulse Wave in Norm: Part 3. International Journal of Biomedicine. 2018;8(4):288-291.

4. Kruglov AG, Utkin VN, Vasilyev AYu, Kruglov AA. Synchronization of Wave Flows of Arterial and Venous Blood and Phases of the Cardiac Cycle. (Part 4). International Journal of Biomedicine. 2019;9(2):106-110

5. Kruglov AG, Utkin VN, Vasilyev AYu, Kruglov AA. Regulatory Synchronization of Hemodynamics of the Heart and Brain in Norm. International Journal of Biomedicine. 2019;9(4):281-286.

6. Kruglov AG, Utkin VN, Vasilyev AYu, Sherman VA. Human Homeostatic Control Matrix in Norm. International Journal of Biomedicine. 2016;6(3):184-9.

7. Kruglov AG, Gebel GYa, Vasilyev AYu. Impact of IntraExtracranial Hemodynamics on Cerebral Ischemia by Arterial Hypertension (Part 1-2). Int J Biomed. 2012;2(2):96-101.

8. Kruglov AG, Gebel GYa, Vasilyev AYu, Sherman VA. Dynamics Networks of Human Homeostatic Control in Norm (Part 2). International Journal of Biomedicine. 2016;6(3):179-183. 